\title{
Casimir effect between moving branes
}

\author{
Antonio L. Maroto* \\ Physics Department, Stanford University, \\ Stanford CA 94305-4060, USA \\ and \\ Departamento de Física Teórica \\ Universidad Complutense de Madrid \\ 28040 Madrid, Spain
}

\begin{abstract}
We consider a supersymmetric model with a single matter supermultiplet in a five-dimensional space-time with orbifold compactification along the fifth dimension. The boundary conditions on the two orbifold planes are chosen in such a way that supersymmetry remains unbroken on the boundaries. We calculate the vacuum energy-momentum tensor in a configuration in which the boundary branes are moving with constant velocity. The results show that the contribution from fermions cancels that of bosons only in the static limit, but in general a velocity-dependent Casimir energy arises between the branes. We relate this effect to the particle production due to the branes motion and finally we discuss some cosmological consequences.
\end{abstract}

PACS numbers: $11.10 . \mathrm{Kk}, 98.80 . \mathrm{Cq}$

*E-mail: maroto@fis.ucm.es 


\section{Introduction}

The presence of non-trivial boundary conditions in quantum field theory leads to interesting phenomena such as the shift in the zero-point energy with respect to the unbounded space configuration [1]. In the case of electromagnetic fields between perfectly conducting parallel plates, such shift has been observed as an atractive force acting on the plates (see [2] for a recent measurement). Since the original work of Casimir, this effect has been studied in different contexts, thus for instance, it plays a fundamental role in hadron physics, in the so-called bag model in which quarks and gluons are confined inside a spherical shell and the corresponding Casimir energy contributes to the total hadron mass. Also in higher-dimensional theories, the stability of the compactified extra dimensions depends crucially on this effect. Other applications can be found in condensed matter physics and atomic or molecular physics (for a review see [3]).

More recently, the increasing interest in the construction of cosmological models based on string or M theory has focused on the so-called brane-world scenario, in which our universe is understood as a 3-brane living in a higher dimensional space-time. A particularly interesting model appears in the low-energy regime of M-theory described by eleven dimensional supergravity [4]. After compactification of six dimensions in a Calabi-Yau manifold, we are left with a five-dimensional model in which matter fields live on the two boundary branes arising after $S^{1} / Z_{2}$-orbifolding the fifth dimension. In this context, the Casimir energy arising between the two static boundaries has been computed in $[5,6]$, in the first of these two works, the backreaction on the geometry was taken into account. The same problem has been considered in five-dimensional anti-deSitter space in [7]. The recently proposed ekpyrotic (cyclic) model of the universe [8] is also based on this framework in which the motion and collision of two such branes is responsible for the Big-Bang of the standard cosmology.

In this paper we are interested in studying the possible effects of the Casimir energy in an scenario like the one mentioned before in which two branes are moving towards each other. The complete analysis of the problem is in general too involved to obtain explicit analytic results and, for that reason, we will consider a simplified model in which the two branes are moving with constant relative velocity and they are perfectly flat, ignoring possible gravitational effects. In any realistic model of a brane collision process it will be necessary to consider the acceleration and the brane curvature [9], but the present analysis would be the first (velocity-dependent) correction to the flat static case. We will also consider a simple global supersymmetric model in five dimensions with a single matter hypermultiplet instead of working with the full supergravity lagrangian in eleven dimensions. Despite these approximations the results will shed light on the velocity dependence of the Casimir energy and therefore on the stability of the branes system. We will be interested also in the role of supersymmetry in the cancellation of the Casimir energy in the moving case. For that purpose we will need to extend previous studies of the dynamical Casimir effect [10] to fermionic fields. In fact we will show that only in the static limit the contribution from fermions cancels that of bosons (as expected from supersymmetry), but in general a velocity dependent Casimir energy arises between the branes.

The paper is organized as follows, in Section 2, we introduce the five-dimensional super- 
symmetric model and set the boundary conditions for the different fields. In Section 3 we compute the scalar contribution to the Casimir stress tensor using the image method for Green functions for arbitrary value of the brane velocity. In Section 4, we perform a similar calculation but with the Bogolyubov transformations technique in the low-velocity regime. Section 5 is devoted to the fermionic contribution, and finally Section 6 contains a discussion on the possible cosmological consequences, paying special attention to the ekpyrotic (cyclic) model of the universe.

\section{Supersymmetric model Lagrangian in 5-D}

Let us consider the simplest globally supersymmetric model in 5-D. A five-dimensional off-shell hypermultiplet consists of two complex scalar fields, which we denote by $\phi_{1}$ and $\phi_{2}$, one fourcomponent Dirac spinor $\psi$ and two auxiliary complex scalars fields $F$ and $G$ [11, 6, 12]. The smallest representation of the Clifford algebra in five dimensions is four dimensional and is given by: $\gamma^{A}=\left\{\gamma^{0}, \gamma^{1}, \gamma^{2}, \gamma^{3}, \gamma^{4}\right\}$ where $\gamma^{4}=i \gamma^{5}$ with the usual definition $\gamma^{5}=i \gamma^{0} \gamma^{1} \gamma^{2} \gamma^{3}$. With this definition we have $\left\{\gamma^{A}, \gamma^{B}\right\}=2 \eta^{A B}$ where $\eta^{A B}=\operatorname{diag}(+,-,-,-,-)$. As a consequence the chirallity operator $\gamma^{0} \gamma^{1} \gamma^{2} \gamma^{3} \gamma^{4}=\mathbf{1}$ is trivial and it is not possible to define chiral fermions in five dimensions.

The simplest supersymmetric action corresponds to massless non-interacting fields and is given by:

$$
S=\int d^{5} x\left(\eta^{A B} \partial_{A} \phi_{1}^{*} \partial_{B} \phi_{1}+\eta^{A B} \partial_{A} \phi_{2}^{*} \partial_{B} \phi_{2}+i \bar{\psi} \gamma^{A} \partial_{A} \psi+|F|^{2}+|G|^{2}\right)
$$

As commented in the introduction, we will assume that the fifth dimension is compactified on the orbifold $S^{1} / Z_{2}$, that is, the fifth dimension is just an interval $[0, L]$, with two parallel boundary planes at $x^{4}=0, L$. In fact, in order to perform the orbifold projection, we need to assign $Z_{2}$ parities $\eta= \pm 1$ to the different fields in such a way that $\Phi\left(x^{\mu}, x^{4}\right)=\eta \Phi\left(x^{\mu},-x^{4}\right)$ where $\Phi$ is any of the hypermultiplet components. A consistent assignment which respects $N=1$ supersymmetry on the boundaries is given in Table 1 (see [13,6]), where we have used the definitions $\psi_{L}=P_{L} \psi=\frac{1-\gamma^{5}}{2} \psi$ and $\psi_{R}=P_{R} \psi=\frac{1+\gamma^{5}}{2} \psi$.

For an $S^{1}$ compactification with radius $R$, the Fourier expansions for the fields with different $Z_{2}$ parities become:

$$
\begin{aligned}
& \Phi_{+}\left(x^{\mu}, x^{4}\right)=\sum_{n=0}^{\infty} \cos \left(n x^{4} / R\right) \Phi_{n}\left(x^{\mu}\right) \\
& \Phi_{-}\left(x^{\mu}, x^{4}\right)=\sum_{n=1}^{\infty} \sin \left(n x^{4} / R\right) \Phi_{n}\left(x^{\mu}\right)
\end{aligned}
$$

We see that even-parity fields satisfy Neumann conditions on the boundary planes, whereas odd-parity fields satisfy Dirichlet conditions i.e. for $L=\pi R$ we have:

$$
\begin{aligned}
\left.\partial_{4} \Phi_{+}\left(x^{\mu}, x^{4}\right)\right|_{x^{4}=0, L} & =0 \\
\left.\Phi_{-}\left(x^{\mu}, x^{4}\right)\right|_{x^{4}=0, L} & =0 .
\end{aligned}
$$




\begin{tabular}{|c|c|}
\hline $\begin{array}{c}\eta=1 \\
\text { (Neumann) }\end{array}$ & $\begin{array}{c}\eta=-1 \\
\text { (Dirichlet) }\end{array}$ \\
\hline$\phi_{1}$ & $\phi_{2}$ \\
\hline$\psi_{R}$ & $\psi_{L}$ \\
\hline$F$ & $G$ \\
\hline
\end{tabular}

Table 1: Parity assignments for the model in (1)

If supersymmetry remains unbroken we expect that the contribution from fermions cancels that of bosons in the vacuum energy-momentum tensor. As we will see this is the case when the boundaries are static, however here we will consider a more general situation in which the boundaries are moving.

\section{Scalar contribution}

Let us write the classical energy-momentum tensor for massless real scalars in a five-dimensional Minkowski background:

$$
\begin{aligned}
T_{A B}^{S} & =(1-2 \xi) \phi_{, A} \phi_{, B}+\left(2 \xi-\frac{1}{2}\right) g_{A B} g^{C D} \phi_{, C} \phi_{, D} \\
& -2 \xi \phi, A B \phi+\frac{2}{5} \eta_{A B} \phi \square \phi
\end{aligned}
$$

where for generality we have considered arbitrary non-minimal couplings in the scalar lagrangian $\xi R \phi^{2}$, which vanish in flat space but however have a non-vanishing contribution to the energymomentum tensor. The particular case $\xi=3 / 16$ corresponds to the conformal coupling in five dimensions

In order to calculate the vacuum expectation value of the energy-momentum tensor we will use the Green's function method. With that purpose we introduce the so called Hadamard function:

$$
D^{(1)}(x, y)=\langle 0|\phi(x) \phi(y)+\phi(y) \phi(x)| 0\rangle
$$

where $|0\rangle$ denotes the usual Minkowski vacuum state built out of the plane-wave solutions of the free equations of motion written in cartesian coordinates. It is possible to write the vacuum expectation value of $T_{A B}$ in cartesian coordinates as:

$$
\left\langle 0\left|T_{A B}^{S}\right| 0\right\rangle=\lim _{x^{\prime} \rightarrow x, y^{\prime} \rightarrow x} \frac{1}{2}\left((1-2 \xi) \partial_{A}^{x^{\prime}} \partial_{B}^{y^{\prime}}+\left(2 \xi-\frac{1}{2}\right) \eta_{A B} \eta^{C D} \partial_{C}^{x^{\prime}} \partial_{D}^{y^{\prime}}-2 \xi \partial_{A}^{x^{\prime}} \partial_{B}^{x^{\prime}}\right) D^{(1)}\left(x^{\prime}, y^{\prime}\right)
$$


where we have used $\square^{x^{\prime}} D^{(1)}\left(x^{\prime}, y^{\prime}\right)=0$. In the simplest case, i.e., unbounded Minkowski space, the Hadamard function can be obtained from the mode expansion of the quantum fields and it is given by:

$$
\begin{aligned}
D^{(1)}(x, y) & =\int \frac{d^{4} p}{2 p_{0}}\left(e^{i p(x-y)}+e^{i p(y-x)}\right) \\
& =\frac{1}{4 \pi^{2}} \frac{\theta(\sigma(x, y))}{\sigma(x, y)^{3 / 2}}
\end{aligned}
$$

where $\sigma(x, y)=-\eta_{A B}\left(x^{A}-y^{A}\right)\left(x^{B}-y^{B}\right), \theta(\sigma(x, y))$ is the step function and $d^{4} p$ is the integration measure for the spatial components of the five-momentum $p_{A}$.

We will first consider the case in which the two branes at $x^{4}=0, L$ are fixed. The Hadamard functions satisfying Dirchlet and Neumann conditions can be calculated by the image method (see for example [13]). According to the principle of mirror reflection such functions can be obtained as a sum over an infinite number of the free functions in (7) evaluated at the image points $x_{n}^{\prime}$. They can be written as:

$$
D_{D / N}^{(1)}\left(x, x^{\prime}\right)=\sum_{n=-\infty}^{\infty}\left(D^{(1)}\left(x, x_{n+}^{\prime}\right) \mp D^{(1)}\left(x, x_{n-}^{\prime}\right)\right)
$$

where the minus sign corresponds to the Dirichlet function and the plus sign to the Neumann one. The image points are given by:

$$
x_{n \pm}^{\prime}=\left(t^{\prime}, x^{\prime 1}, x^{\prime 2}, x^{\prime 3}, \pm x^{4}-2 n L\right)
$$

These functions satisfy the corresponding boundary conditions given in (3). Since in our model, one complex scalar field satisfies Dirichlet conditions and the other one Neumann conditions, the total scalar energy-momentum tensor will be given by the sum of the two kinds of contributions, i.e.:

$$
\begin{aligned}
& \left\langle 0\left|T_{A B}^{S}\right| 0\right\rangle=\lim _{x^{\prime}, y^{\prime} \rightarrow x} 2\left((1-2 \xi) \partial_{A}^{x^{\prime}} \partial_{B}^{y^{\prime}}-2 \xi \partial_{A}^{x^{\prime}} \partial_{B}^{x^{\prime}}\right. \\
& \left.+\left(2 \xi-\frac{1}{2}\right) \eta_{A B} \eta^{C D} \partial_{C}^{x^{\prime}} \partial_{D}^{y^{\prime}}\right) \sum_{n=-\infty}^{\infty} D^{(1)}\left(x^{\prime}, y_{n+}^{\prime}\right)
\end{aligned}
$$

The expression in (10) is divergent in the coincidence limit, the divergent contribution coming from the $n=0$ term which is nothing but the unbounded space vacuum energy-momentum tensor. Thus we can define the renormalized vacuum expectation value substracting this free space term:

$$
\left\langle 0\left|T_{A B}\right| 0\right\rangle_{\text {ren }}=\left\langle 0\left|T_{A B}\right| 0\right\rangle-\left\langle 0\left|T_{A B}\right| 0\right\rangle_{n=0}
$$

The final result does not depend on the non-minimal parameter $\xi$ and is given by the well-known expression:

$$
\left\langle 0\left|T_{A B}^{S}\right| 0\right\rangle_{\text {ren }}^{\text {static }}=-\frac{3 \zeta(5)}{32 \pi^{2} L^{5}}\left(\begin{array}{ccc}
1 & 0 & 0 \\
0 & -1 & 0 \\
0 & 0 & 4
\end{array}\right)
$$


Let us consider next the case in which the 3 -brane at $x^{4}=0$ is fixed whereas the other one is moving with constant velocity $v$ in the fifth dimension. In order to obtain the image points it is easier to work in a coordinate system in which the branes are fixed, but the geometry is contracting (expanding) along the fifth dimension [14]. Thus, let us consider the new coordinates $\left(\tau, x^{1}, x^{2}, x^{3}, \chi\right)$ defined as:

$$
x^{0}=\tau \cosh (\chi), \quad x^{4}=\tau \sinh (\chi)
$$

In these ccordinates the branes positions are given simply by $\chi=0$ and $\chi=\chi_{0}$. Notice that curves with constant fifth coordinate $\chi=\chi_{0}$ describe brane motions with a constant velocity $v=\tanh \left(\chi_{0}\right)$. The corresponding metric tensor reads:

$$
d s^{2}=d \tau^{2}-\left(d x^{1}\right)^{2}-\left(d x^{2}\right)^{2}-\left(d x^{3}\right)^{2}-\tau^{2} d \chi^{2}
$$

which is nothing but the Milne metric in five dimensions. This metric can describe the portions of Minkowski space-time with either $x^{0}>0$ or $x^{0}<0$. We will use indices $A, B, C, \ldots=$ $0,1,2,3,4$ for Minkowski coordinates and $M, N, P, \ldots=0,1,2,3,4$ for the Milne coordinates. For this metric the only non-vanishing Christoffel symbols are $\hat{\Gamma}_{\chi \chi}^{\tau}=a^{2} \tau, \hat{\Gamma}_{\tau \chi}^{\chi}=\hat{\Gamma}_{\chi \tau}^{\chi}=\frac{1}{\tau}$. In the following, we will use the hat to denote objects written in Milne coordinates. The invariant interval $\sigma\left(x, x^{\prime}\right)$ defined before can be written in the new coordinates as:

$$
\begin{aligned}
\sigma\left(x, x^{\prime}\right) & =-\tau^{2}-\tau^{\prime 2}+2 \tau \tau^{\prime} \cosh \left(\chi-\chi^{\prime}\right)+\left(x^{1}-x^{\prime 1}\right)^{2} \\
& +\left(x^{2}-x^{\prime 2}\right)^{2}+\left(x^{3}-x^{\prime 3}\right)^{2}
\end{aligned}
$$

The Dirichlet and Neumann functions are still given by (8), but with the image points written in the new coordinates, i.e.:

$$
x_{n \pm}^{\prime}=\left(\tau^{\prime}, x^{\prime 1}, x^{\prime 2}, x^{\prime 3}, \pm \chi^{\prime}-2 n \chi_{0}\right)
$$

The boundary conditions satisfied by the Green functions now read:

$$
\begin{aligned}
\left.D_{D}^{(1)}\left(x, x^{\prime}\right)\right|_{\chi, \chi^{\prime}=0, \chi_{0}} & =0, \\
\left.\partial_{\chi} D_{N}^{(1)}\left(x, x^{\prime}\right)\right|_{\chi, \chi^{\prime}=0, \chi_{0}} & =0
\end{aligned}
$$

Using Eqs. (6), (11) and changing to Milne coordinates, we obtain the renormalized expressions for the energy-momentum tensor. In these coordinates the tensors are diagonal and do not depend on the spatial coordinates, however unlike the static case, they do depend on the $\xi$ parameter. Thus for minimal coupling $\xi=0$ we get:

$$
\left\langle 0\left|\hat{T}_{M N}^{S}\right| 0\right\rangle_{\text {ren }}^{\text {mov }}=-\frac{3}{32 \pi^{2}|\tau|^{5}} \sum_{n=1}^{\infty} \frac{1}{\left|\sinh ^{5}\left(n \chi_{0}\right)\right|}\left(\begin{array}{ccc}
1 & 0 & 0 \\
0 & -\frac{3 \cosh \left(2 n \chi_{0}\right)-1}{2} 1 & 0 \\
0 & 0 & 4 \tau^{2}
\end{array}\right)
$$

whose trace is non-vanishing. Notice that when the branes are fixed the result (12) is traceless regardless of the value of the $\xi$ parameter. For the conformal coupling $\xi=3 / 16$, we get:

$$
\left\langle 0\left|\hat{T}_{M N}^{S}\right| 0\right\rangle_{\text {ren }}^{\text {mov }}=-\frac{3}{32 \pi^{2}|\tau|^{5}} \sum_{n=1}^{\infty} \frac{5+3 \cosh \left(2 n \chi_{0}\right)}{8\left|\sinh ^{5}\left(n \chi_{0}\right)\right|}\left(\begin{array}{ccc}
1 & 0 & 0 \\
0 & -1 & 0 \\
0 & 0 & 4 \tau^{2}
\end{array}\right)
$$


which is traceless as expected. These results are valid for arbitrary values of the brane velocity. However, in order to extract an explicit velocity contribution to the Casimir energy-momentum tensor, we will compare them in the non-relativisitic limit $v \ll 1$ with the static result in (12). In Milne coordinates, the proper distance between the two branes is given by $d=\tau \chi_{0}$, thus, in such limit, we get for minimal coupling:

$$
\left\langle 0\left|\hat{T}_{M N}^{S}\right| 0\right\rangle_{\text {ren }}^{\text {mov }} \simeq-\frac{3 \zeta(5)}{32 \pi^{2} d^{5}}\left(\begin{array}{ccc}
1 & 0 & 0 \\
0 & -\mathbf{1} & 0 \\
0 & 0 & 4 \tau^{2}
\end{array}\right)+\frac{5 \zeta(3) v^{2}}{64 \pi^{2} d^{5}}\left(\begin{array}{ccc}
1 & 0 & 0 \\
0 & \frac{13}{5} \mathbf{1} & 0 \\
0 & 0 & 4 \tau^{2}
\end{array}\right)
$$

and for conformal coupling:

$$
\left\langle 0\left|\hat{T}_{M N}^{S}\right| 0\right\rangle_{\text {ren }}^{\text {mov }} \simeq-\frac{3}{32 \pi^{2} d^{5}}\left(\zeta(5)-\frac{\zeta(3)}{12} v^{2}\right)\left(\begin{array}{ccc}
1 & 0 & 0 \\
0 & -\mathbf{1} & 0 \\
0 & 0 & 4 \tau^{2}
\end{array}\right)
$$

The velocity contribution to the Casimir energy is quadratic and positive in the non-relativisitic limit. In the following we will show that such contribution is nothing but the energy density in the form of particles created from the vacuum by the moving boundaries.

\section{Particle production}

Let us consider a quantized real scalar field:

$$
\phi(x)=\sum_{\vec{k}} \sum_{n}\left(a_{\vec{k} n} u_{\vec{k} n}+a_{\vec{k} n}^{\dagger} u_{\vec{k} n}^{*}\right)
$$

The modes $u_{\vec{k} n}$ obeying the Klein-Gordon equation in Milne coordinates are given by:

$$
\begin{array}{cc}
u_{\vec{k} n}^{N}(x)=N f_{k n}(\tau) e^{i \vec{k} \vec{x}} \cos (\nu \chi), & \text { Neumann } \\
u_{\vec{k} n}^{D}(x)=N f_{k n}(\tau) e^{i \vec{k} \vec{x}} \sin (\nu \chi), & \text { Dirichlet }
\end{array}
$$

where the functions $f_{k n}(\tau)$ satisfy the equation:

$$
\left(\hat{\partial}_{0} \hat{\partial}_{0}+k^{2}+\frac{\nu^{2}}{\tau^{2}}+\frac{1}{\tau} \hat{\partial}_{0}\right) f_{k n}(\tau)=0
$$

with $k^{2}=\vec{k}^{2}, \nu=n \pi / \chi_{0}$ and $n$ a positive integer number. The solutions of equation (24) can be written in terms of Hankel functions $f_{k n}=H_{i \nu}^{(1,2)}(k \tau)$ where the $1(2)$ functions behave as positive frequency modes in the $\tau \rightarrow+(-) \infty$ limit. The solutions are normalized with respect to the scalar product:

$$
\left(\phi_{1}, \phi_{2}\right)=-i \int_{V} d^{3} x \int_{0}^{\chi_{0}} d \chi \tau\left(\phi_{1}^{*} \hat{\partial}_{0} \phi_{2}-\hat{\partial}_{0} \phi_{1}^{*} \phi_{2}\right)
$$


where we are working in a box of finite volume $V$ in the ordinary three-space. We will take the continuum limit at the end of the calculations. From the expression above, we can obtain the normalization constant $N=\sqrt{\frac{\pi}{2 V \chi_{0}}} e^{-\nu \pi / 2}$.

Because of the moving boundaries, a given solution which behaves as positive frequency at a given time will become a linear superposition of positive and negative frequency modes at any later time. This implies that the initial vacuum state will contain particles as seen by an observer at a later time. Thus consider the solutions at a given time for the static problem with a fixed brane separation $L$. The positive frequency modes satisfying the different boundary conditions are given by:

$$
\begin{array}{lr}
v_{\vec{k} n}^{N}=N e^{-i \omega t+i \vec{k} \vec{x}} \cos (q z), & \text { Neumann } \\
v_{\vec{k} n}^{D}=N e^{-i \omega t+i \vec{k} \vec{x}} \sin (q z), & \text { Dirichlet }
\end{array}
$$

where $q=n \pi / L, \omega^{2}=k^{2}+q^{2}$ and $N=(L V \omega)^{-1 / 2}$. These modes will define a different Fock space which can be built out of the corresponding creation and annhilation operators which we will denote by $b_{\vec{k} n}, b_{\vec{k} n}^{\dagger}$. Thus, for either the real or the imaginary part of our scalar fields we have:

$$
\phi(x)=\sum_{\vec{k}} \sum_{n}\left(b_{\vec{k} n} v_{\vec{k} n}+b_{\vec{k} n}^{\dagger} v_{\vec{k} n}^{*}\right)
$$

The two sets of operators are related in general by the following Bogolyubov transformation $[15]$ :

$$
b_{\vec{k} n}=\sum_{\vec{k}^{\prime}} \sum_{n^{\prime}}\left(\alpha_{\vec{k} \vec{k}^{\prime} n n^{\prime}} a_{\vec{k}^{\prime} n^{\prime}}+\beta_{\vec{k} \vec{k}^{\prime} n n^{\prime}}^{*} a_{\vec{k}^{\prime} n^{\prime}}^{\dagger}\right)
$$

The Bogolyubov coefficients relating both solutions are given by: $\beta_{\vec{k} \vec{k}^{\prime}, n n^{\prime}}=\left(u_{\vec{k} n}, v_{\vec{k}^{\prime} n^{\prime}}^{*}\right)$. Following [14], we are interested in the calculation of the total number of particles created by the brane motion in the non-relativisitic limit $\chi_{0} \ll 1$, i.e. for large values of $\nu$. In that limit, we can use the large-order expansion of Hankel functions in the calculation of the Bogolyubov coefficients. In the present case it is enough with the first term in the expansion which is given by $[16]$ :

$$
H_{i \nu}^{(1)}(k \tau) \simeq \sqrt{\frac{2}{\pi}} \frac{1}{\left(\nu^{2}+k^{2} \tau^{2}\right)^{1 / 4}} \exp \left(\frac{\nu \pi}{2}+i\left(\nu^{2}+k^{2} \tau^{2}\right)^{1 / 2}-i \nu \operatorname{arcsinh}\left(\frac{\nu}{k \tau}\right)-i \frac{\pi}{4}\right)
$$

This expression is valid for large $\tau$, i.e. large brane separations and satisfies the equation of motion up to terms of $\mathcal{O}\left(\nu^{-2}\right)$. In the non-relativistic limit, we have $t=x^{0}=\tau+\mathcal{O}\left(\chi^{2}\right)$, $z=\tau \chi+\mathcal{O}\left(\chi^{3}\right)$ and $v=\chi_{0}+\mathcal{O}\left(\chi_{0}^{3}\right)$, so that we can write $L=\tau \chi_{0}+\mathcal{O}\left(\chi_{0}^{3}\right)$ and $q=\nu / \tau+\mathcal{O}\left(\chi_{0}^{2}\right)$ so that $\omega^{2}=k^{2}+\nu^{2} / \tau^{2}+\mathcal{O}\left(\chi_{0}^{2}\right)$. Therefore for the Bogolyubov coefficients relating Neumann solutions we get:

$$
\beta_{\vec{k} \vec{k}^{\prime} n n^{\prime}} \simeq-i \int_{V} d^{3} x \int_{0}^{L} d z \cos \left(\frac{n^{\prime} \pi z}{L}\right) \cos \left(\frac{n \pi \chi}{\chi_{0}}\right) \frac{e^{i\left(\vec{k}^{\prime}-\vec{k}\right) \vec{x}}}{2 \chi_{0}} \frac{k^{2} \tau}{\left(\nu^{2}+k^{2} \tau^{2}\right)^{3 / 2}} e^{i \Phi(\nu, k, \tau)}
$$




$$
=-i \delta_{\vec{k} \overrightarrow{k^{\prime}}} \delta_{n n^{\prime}} \frac{k^{2} \tau^{2}}{4\left(\nu^{2}+k^{2} \tau^{2}\right)^{3 / 2}} e^{i \Phi(\nu, k, \tau)}+\mathcal{O}\left(\chi_{0}^{0}\right)
$$

where the phase is given by:

$$
\Phi(\nu, k, \tau)=2 \omega \tau-\nu \operatorname{arcsinh}\left(\frac{\nu}{k \tau}\right)+\frac{\pi}{4}
$$

and the same results is obtained for Dirichlet solutions, whereas mixed Dirichlet-Neumann coefficients vanish. Defining $\beta_{\vec{k} \vec{k}^{\prime} n n^{\prime}}=\beta_{\vec{k} n} \delta_{k k^{\prime}} \delta_{n n^{\prime}}$, the total energy-density of the particles created for the scalar matter content in our model with two complex scalar fields is:

$$
\rho_{S}=\frac{4}{L} \sum_{n=0}^{\infty} \int \frac{d^{3} k}{(2 \pi)^{3}} \omega\left|\beta_{\vec{k} n}\right|^{2} \simeq \frac{4}{L} \sum_{n=0}^{\infty} \int \frac{d^{3} k}{(2 \pi)^{3}} \frac{k^{4} \tau^{4} \omega}{16\left(\nu^{2}+k^{2} \tau^{2}\right)^{3}}
$$

where we have already taken the continuum limit $V \rightarrow \infty$. This is a divergent expression which can be renormalized using the zeta-function method [17]. By means of a change of variables, we can write the above expression as:

$$
\rho_{S} \simeq \frac{1}{8 \pi^{2} \chi_{0} \tau^{5}} \sum_{n=0}^{\infty} \int_{0}^{\infty} d x \frac{x^{6}}{\left(x^{2}+\nu^{2}\right)^{5 / 2}}=\frac{1}{16 \pi^{2} \tau^{5} \chi_{0}} B\left(\frac{7}{2},-1\right) \sum_{n=0}^{\infty}\left(\frac{n \pi}{\chi_{0}}\right)^{2}
$$

where $B(p, q)=\Gamma(p) \Gamma(q) / \Gamma(p+q)$ is the beta function and $\sum_{n} n^{-s}=\zeta(s)$ is the Riemann zeta function. We can cancel the divergent contributions from the integral and the series by using the analytical continuation of the relation: $\zeta(s)=\Gamma\left(\frac{1-s}{2}\right) \pi^{s-1 / 2} \zeta(1-s) / \Gamma\left(\frac{s}{2}\right)$, we obtain the following renormalized result:

$$
\rho_{S} \simeq \frac{5}{64 \pi^{2} \tau^{5} \chi_{0}^{5}} \zeta(3) v^{2}
$$

which agrees with the first correction to the static Casimir energy in (20). We see that this velocity correction can be understood as the contribution from the particles produced by the non-adiabatic motion of the branes [14].

\section{Fermionic contribution}

Although the fermionic Casimir effect has been considered previously in different contexts, only a few results in the case with moving boundaries can be found in the literature [18]. In this section we will consider the problem in our five dimensional model.

The fermionic energy-momentum tensor for massless fermions in five dimensions can be written in Minkowski coordinates as:

$$
T_{A B}^{F}=\frac{i}{4}\left(\bar{\psi} \gamma_{A} \partial_{B} \psi+\bar{\psi} \gamma_{B} \partial_{A} \psi-\partial_{A} \bar{\psi} \gamma_{B} \psi-\partial_{B} \bar{\psi} \gamma_{A} \psi\right)
$$


Following similar steps to the scalar case, we will compute the corresponding vacuum expectation value, in the case in which the two branes are fixed, by means of the image method. The fermionic Hadamard function can be written as:

$$
\mathcal{D}_{a b}^{(1)}(x, y)=\left\langle 0\left|\bar{\psi}_{b}(y) \psi_{a}(x)-\bar{\psi}_{b}(x) \psi_{a}(y)\right| 0\right\rangle
$$

where $a, b$ are spinor indices. From (35), the vacuum expectation value of $T_{A B}^{F}$ can be written in Minkowski space-time in terms of the Hadamard function as:

$$
\left\langle 0\left|T_{A B}^{F}\right| 0\right\rangle=\lim _{x^{\prime} \rightarrow x, y^{\prime} \rightarrow x} \frac{i}{4}\left(\left(\gamma_{A}\right)_{b a} \partial_{B}^{x^{\prime}}+\left(\gamma_{B}\right)_{b a} \partial_{A}^{x^{\prime}}\right) \mathcal{D}_{a b}^{(1)}\left(x^{\prime}, y^{\prime}\right)
$$

In the simplest case with no boundary conditions and in Minkowski coordinates, the Hadamard function is given in terms of the scalar function (7) as $\mathcal{D}_{a b}^{(1)}(x, y)=-i \gamma_{a b}^{A} \partial_{A}^{x} D^{(1)}(x, y)$. In the case with fixed boundaries, the different fermionic components satisfy (3) and the Hadamard function obeying these boundary conditions can be written as:

$$
\begin{aligned}
\mathcal{D}^{(1)}(x, y) & =P_{L} \mathcal{D}_{D D}^{(1)}(x, y) P_{R}+P_{R} \mathcal{D}_{N N}^{(1)}(x, y) P_{L} \\
& +P_{L} \mathcal{D}_{D N}^{(1)}(x, y) P_{L}+P_{R} \mathcal{D}_{N D}^{(1)}(x, y) P_{R}
\end{aligned}
$$

With these relations, we obtain from (37) the following expression for the energy-momentum tensor between the fixed branes:

$$
\begin{aligned}
\left\langle 0\left|T_{A B}^{F}\right| 0\right\rangle & =\lim _{x^{\prime} \rightarrow x, y^{\prime} \rightarrow x} \frac{1}{4} \operatorname{tr}\left(\partial_{B}^{x^{\prime}} \mathcal{D}_{D D}^{(1)}\left(x^{\prime}, y^{\prime}\right) P_{R} \gamma_{A} P_{L}+\partial_{B}^{x^{\prime}} \mathcal{D}_{N N}^{(1)}\left(x^{\prime}, y^{\prime}\right) P_{L} \gamma_{A} P_{R}\right. \\
& \left.+\partial_{B}^{x^{\prime}} \mathcal{D}_{D N}^{(1)}\left(x^{\prime}, y^{\prime}\right) P_{L} \gamma_{A} P_{L}+\nabla_{B}^{x^{\prime}} \mathcal{D}_{N D}^{(1)}\left(x^{\prime}, y^{\prime}\right) P_{R} \gamma_{A} P_{R}\right)+(A \leftrightarrow B)
\end{aligned}
$$

In order to calculate the explicit expression of the different components of the Hadamard function in (38), we need to know the mode expansion of the Dirac field:

$$
\psi(x)=\sum_{\vec{k} n \alpha}\left(b_{\vec{k} n \alpha} v_{\vec{k} n \alpha}^{+}(x)+d_{\vec{k} n \alpha}^{\dagger} v_{\vec{k} n \alpha}^{-}(x)\right)
$$

where we have labelled the states by $\alpha= \pm 1 / 2$, according to the value of the spin projection along the ordinary three-momentum on the brane. The positive and negative frequency solutions of the Dirac equation in Minkowski coordinates are given by:

$$
v_{\vec{k} n \alpha}^{ \pm}(x)=\frac{1}{\sqrt{2 L V \omega(\omega \pm q)}} e^{\mp i \omega t} e^{i \vec{k} \vec{x}}\left(\begin{array}{c}
\sin (q z) U_{\alpha} \\
\cos (q z) U_{\alpha}
\end{array}\right)
$$

where the two-component spinors with the appropriate helicities are given by: $U_{1 / 2}^{T}=(1,0)$ and $U_{-1 / 2}^{T}=(0,1)$ and the component of the momentum in the fifth dimension $q$ is defined as in the scalar case with fixed boundaries. The modes are normalized with respect to the scalar product:

$$
\left(\psi_{1}, \psi_{2}\right)=\int d^{4} x \sqrt{g_{0}} \bar{\psi}_{1} \hat{\gamma}_{0} \psi_{2}
$$


where $g_{0}$ denotes the determinant of the induced metric on the hypersurfaces of constant time. In the calculation of the energy-momentum tensor in (39) only derivatives of the Green functions are relevant, thus, making use of the the equations of motion, $\not \partial^{x} \mathcal{D}^{(1)}(x, y)=0$ and acting with the projectors we get:

$$
\gamma^{4} \partial_{4}^{x} P_{R} \mathcal{D}_{N D}^{(1)}(x, y) P_{R}=-\gamma^{\mu} \partial_{\mu}^{x} P_{L} \mathcal{D}_{D D}^{(1)}(x, y) P_{R}
$$

and a similar expression is obtained for the $N N$ function. Tracelessness and conservation determine a diagonal form of the energy-momentum tensor in the Minkowski background. Accordingly the knowledge of the $D D$ and $N N$ functions together with Eq. (43) is sufficient to compute the complete tensor. Inserting the explicit mode expansion (40) into (38), it can be shown that such functions are given by:

$$
P_{L} \mathcal{D}_{D D}^{(1)}(x, y) P_{R}=-i P_{L} \not \partial^{x} D_{D}^{(1)}(x, y) P_{R}
$$

where $D_{D}^{(1)}(x, y)$ is the scalar function given in (8) and a similar expression is obtained for the $N N$ function. From (39) we finally get:

$$
\left\langle 0\left|T_{A B}^{F}\right| 0\right\rangle_{\text {ren }}^{\text {static }}=2 \lim _{x^{\prime} \rightarrow x, y^{\prime} \rightarrow x} \sum_{n=-\infty}^{\infty} \partial_{A}^{x^{\prime}} \partial_{A}^{x^{\prime}} D^{(1)}\left(x^{\prime}, y_{n+}^{\prime}\right) \delta_{A B}=\frac{3 \zeta(5)}{32 \pi^{2} L^{5}}\left(\begin{array}{ccc}
1 & 0 & 0 \\
0 & -1 & 0 \\
0 & 0 & 4
\end{array}\right)
$$

which agrees with the static scalar case (12) but with opposite sign. Accordingly, and as expected from supersymmetry, for static branes the total energy-momentum vanishes.

The case in which one of the branes is moving cannot be computed easily with the image method, so that in the following we will use the Bogolyubov transformations technique. Let us then consider again Milne coordinates. We introduce the following expression for the vielbein $e_{M}^{A}$ and the inverse vielbein $\hat{e}_{A}^{M}$ corresponding to the Milne metric in (14): $\hat{e}_{\nu}^{\mu}=e_{\nu}^{\mu}=\delta_{\nu}^{\mu}$ with $\mu=0, . ., 3$, and $e_{4}^{4}=\tau, \hat{e}_{4}^{4}=1 / \tau$. The curved gamma matrices are defined as usual by $\hat{\gamma}^{M}=\hat{e}_{A}^{M} \gamma^{A}$. The Dirac equation in these coordinates reads:

$$
i \hat{D D} u=\left(i \gamma^{\mu} \hat{\partial}_{\mu}+\frac{i}{\tau} \gamma^{4} \hat{\partial}_{4}+\frac{i}{2 \tau} \gamma^{0}\right) u=0
$$

We look for solutions in the form $u_{\vec{k} n \alpha}^{ \pm}=i \not \hat{D} \hat{\psi}_{\vec{k} n \alpha}^{ \pm}$with:

$$
\hat{\psi}_{\vec{k} n \alpha}^{ \pm}(x)=N f_{\vec{k} n}^{ \pm}(\tau) e^{i \vec{k} \vec{x}}\left(\begin{array}{c}
\sin (\nu \chi) U_{\alpha} \\
\cos (\nu \chi) U_{\alpha}
\end{array}\right)
$$

where \pm index denotes positive or negative frequency and again the fifth component of the momentum $\nu$ is defined as in the scalar case with moving boundaries. With these definitions it can be seen that the spinor $u_{\vec{k} n \alpha}^{ \pm}$obeys the correct boundary conditions, i.e.:

$$
\begin{aligned}
\left.P_{L} u_{\vec{k} n \alpha}^{ \pm}\right|_{\chi=0, \chi_{0}} & =0, \\
\left.\partial_{\chi} P_{R} u_{\vec{k} n \alpha}^{ \pm}\right|_{\chi=0, \chi_{0}} & =0
\end{aligned}
$$


The function $f_{\vec{k} n}^{ \pm}(\tau)$ satisfies the equation:

$$
f_{\vec{k} n}^{ \pm \prime \prime}(\tau)+\left(k^{2}+\frac{\nu^{2}}{\tau^{2}}-\frac{1}{4 \tau^{2}}+\frac{i \nu}{\tau^{2}}\right) f_{\vec{k} n}^{ \pm}(\tau)+\frac{1}{\tau} f_{\vec{k} n}^{ \pm \prime}(\tau)=0
$$

The corresponding solutions are given by Hankel functions $f_{\vec{k} n}^{+}(\tau)=H_{i \nu-1 / 2}^{(1)}(k \tau)$ and $f_{\vec{k} n}^{-}(\tau)=$ $H_{i \nu-1 / 2}^{(2)}(k \tau)$. In the non-relativistic limit $\nu \gg 1$ the asymptotic expansion of the positive frequency Hankel function reads [16]:

$$
\begin{aligned}
H_{i \nu-1 / 2}^{(1)}(k \tau) & \simeq \sqrt{\frac{2}{\pi}} \frac{1}{\left(k^{2} \tau^{2}+\nu^{2}\right)^{1 / 4}}\left(1-\frac{i \nu}{4\left(k^{2} \tau^{2}+\nu^{2}\right)}\right) \\
& \times \exp \left(i\left(k^{2} \tau^{2}+\nu^{2}\right)^{1 / 2}+\frac{\nu \pi}{2}-\left(i \nu-\frac{1}{2}\right) \operatorname{arcsinh}\left(\frac{\nu}{k \tau}\right)\right)
\end{aligned}
$$

This expansion satisfies Eq.(46) up to $\mathcal{O}\left(\nu^{-2}\right)$. The normalization constant can be obtained from the scalar product (42) and is given by: $N=\frac{1}{2} e^{-\pi \nu / 2} \sqrt{\frac{\pi}{k V \chi_{0}}}$. Following similar steps to the scalar case, we calculate the number of fermions produced by means of the Bogolyubov coeffcient $\beta_{\vec{k} \vec{k}^{\prime}, n n^{\prime}, \alpha \alpha^{\prime}}=\left(u_{\vec{k} n \alpha}^{+}, v_{\overrightarrow{k^{\prime} n^{\prime} \alpha^{\prime}}}^{-}\right)$. After a lengthy calculation we obtain $\beta_{\vec{k} \vec{k}^{\prime}, n n^{\prime}, \alpha \alpha^{\prime}}=\beta_{\vec{k} n \alpha} \delta_{\vec{k} \vec{k}^{\prime}} \delta_{n n^{\prime}} \delta_{\alpha \alpha^{\prime}}$ with $\beta_{\vec{k} n \alpha}=0$ to the lowest order i.e. $\mathcal{O}\left(\nu^{0}\right)$ and

$$
\begin{aligned}
\beta_{\vec{k} n \alpha} & \simeq \frac{i}{4 k \nu\left(k^{2}+\frac{\nu^{2}}{\tau^{2}}\right)^{3 / 2}}\left(\frac{\nu}{\tau}\left(k^{2}+\frac{\nu^{2}}{\tau^{2}}\right)^{3 / 2}+\frac{\nu^{2}}{\tau^{2}}\left(k^{2}+\frac{\nu^{2}}{\tau^{2}}\right)+\frac{\nu^{2} k^{2}}{\tau^{2}}\right) \\
& \times \exp \left(2 i \omega \tau-i \nu \operatorname{arcsinh}\left(\frac{\nu}{k \tau}\right)+i \frac{\pi}{4}\right)
\end{aligned}
$$

including the first order $\left(\mathcal{O}\left(\nu^{-1}\right)\right)$ correction.

The total energy density to this order is then given by:

$$
\rho_{F}=\frac{2}{L} \sum_{\alpha= \pm 1 / 2} \sum_{n=0}^{\infty} \int \frac{d^{3} k}{(2 \pi)^{3}} \omega\left|\beta_{\vec{k} n \alpha}\right|^{2}
$$

where the factor of two comes from the fact that antifermions are produced in the same amount as fermions. Using again zeta-function regularization to perform the integrals we obtain:

$$
\begin{aligned}
\rho_{F} & \simeq \frac{\pi^{2}}{16 \tau^{4} \chi_{0}^{2} L}\left(B\left(\frac{1}{2},-1\right)+B\left(\frac{1}{2}, 0\right)+B\left(\frac{5}{2}, 0\right)+B\left(\frac{1}{2},-\frac{1}{2}\right)\right. \\
& \left.+B\left(\frac{3}{2},-\frac{1}{2}\right)+B\left(\frac{3}{2}, 0\right)\right) \sum_{n=0}^{\infty} n^{2}=-\frac{9}{64 \pi^{2} \tau^{5} \chi_{0}^{3}} \zeta(3)
\end{aligned}
$$

Finally combining this result with the static contribution in (12) and taking into account that for massless fermions the theory is conformally invariant, the total result for the renormalized energy-momentum tensor up to second order in the velocity is:

$$
\left\langle 0\left|\hat{T}_{M N}^{F}\right| 0\right\rangle_{\text {ren }}^{\text {mov }} \simeq \frac{3}{32 \pi^{2} d^{5}}\left(\zeta(5)-\frac{3 \zeta(3)}{2} v^{2}\right)\left(\begin{array}{ccc}
1 & 0 & 0 \\
0 & -\mathbf{1} & 0 \\
0 & 0 & 4 \tau^{2}
\end{array}\right)
$$


We can compute the total result including minimal scalar and fermion contributions, it is given by:

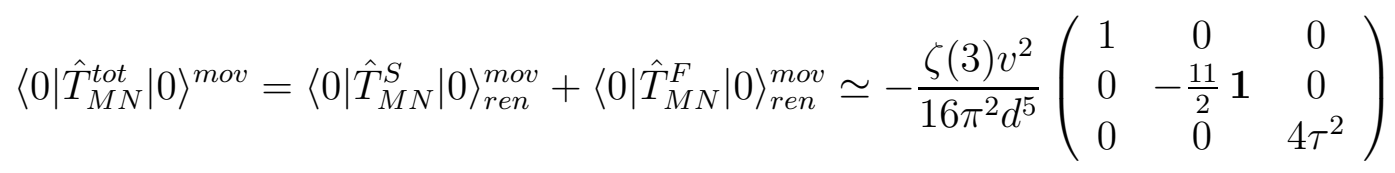

For conformal coupling we get:

$$
\left\langle 0\left|\hat{T}_{M N}^{t o t}\right| 0\right\rangle^{m o v} \simeq-\frac{17 \zeta(3) v^{2}}{128 \pi^{2} d^{5}}\left(\begin{array}{ccc}
1 & 0 & 0 \\
0 & -\mathbf{1} & 0 \\
0 & 0 & 4 \tau^{2}
\end{array}\right)
$$

We see that the result is non-vanishing for $v \neq 0$, i.e. a Casimir effect arises between the branes even though we are using the same kind of boundary conditions which preserved supersymmetry in the static case.

\section{Conclusions and discussion}

In this work we have considered the dynamical Casimir effect in a five dimensional global supersymmetric model between two moving boundary branes. Taking the same kind of boundary conditions as in the static case, the results with moving boundaries are however completely different. In fact a non-vanishing negative Casimir energy is generated both for minimal and conformally coupled scalars. This induces a new type of velocity dependent potential between the branes and the breaking of supersymmetry due to the branes motion.

The presence of this additional energy density could have interesting cosmological effects. Thus for instance, in the context of the ekpyrotic (cyclic) model [8], the universe contracts to a singularity and then reexpands. However it was shown in [19] that ultra-relativistic particles produced near the singularity could disrupt the cyclic evolution provided their energy density reaches the Planckian scale. We have shown that the velocity contribution to the Casimir effect can be interpreted as particle production in the Minkowski vacuum and in fact the energy density is expected to be very high in the limit of small brane separation. The stability of this kind of orbifold singularities has been also studied in [20]. According to their conclusions, the introduction of a single particle between the branes causes the collapse of the universe into a curvature singularity.

Concerning the problem of particle production in a Milne metric, in a recent paper [21], it has been shown that by matching modes through the singularity for a free theory, it is possible to find an appropriate vacuum state in which particles are not produced. However, when including time-dependent interactions, particles are generically produced. We understand that this should be the case in the presence of the moving boundaries, implying that the energymomentum tensor calculated in this work is physical and should backreact on the space-time geometry. (See also [22] for a discussion about the backreaction problem.) 


\section{Acknowledgments}

I am grateful to A. Linde for useful discussions and important suggestions and to C. Herdeiro, S. Hirano and L. Kofman for useful comments and discussions. This work has been partially supported by the CICYT (Spain) project FPA2000-0956. The author also acknowledges support from the Universidad Complutense del Amo Program.

\section{References}

[1] H.B.G. Casimir, Proc. Kon. Nederl. Akad. Wet. 51 (1948) 793

[2] G. Bressi, G. Carugno, R. Onofrio and G. Ruoso, Phys. Rev. Lett. 88, 041804 (2002)

[3] M. Bordag, U.Mohideen and V.M. Mostepanenko, Phys. Rept. 353 1, (2001)

[4] P. Hořava and E. Witten, Nucl. Phys. B460, 506 (1996); ibid., B475, 94 (1996)

[5] M. Fabinger and P. Hořava, Nucl. Phys. B580, 243 (2000)

[6] E.A. Mirabelli and M.E. Peskin, Phys. Rev. D58, 065002 (1998)

[7] S.Nojiri, S. Odintsov and S. Zerbini, Class. Quant. Grav. 17, 4855 (2000); I. Brevik, K. Milton, S. Nojiri and S. Odintsov, Nucl.Phys. B599, 305 (2001)

[8] J. Khoury, B.A. Ovrut, P.J. Steinhardt and N. Turok, Phys.Rev. D64, 123522 (2001); P.J. Steinhardt, N. Turok, Phys.Rev. D65, 126003 (2002)

[9] S. Rasanen, Nucl.Phys. B626, 183 (2002)

[10] M. Bordag, G. Petrov and D. Robashik, Sov. J. Nucl. Phys. 39, 828 (1984); M. Bordag, F.M. Dittes and D. Robashik, Sov. J. Nucl. Phys. 43, 1034 (1986)

[11] P. West, Introduction to Supersymmetry and Supergravity, World Scientific, (1986)

[12] A. Pomarol and M. Quirós, Phys.Lett. B438, 255 (1998)

[13] Y. Igarashi, Phys.Rev.D30, 1812 (1984); Y. Igarashi and T. Nonoyama, Prog.Theor.Phys. 77, 427 (1987)

[14] R. Jáuregui, C. Villareal and S. Hacyan, Phys.Rev. A52, 594 (1995); R. Jáuregui, C. Villareal and S. Hacyan, Mod. Phys. Lett. A10, 619 (1995)

[15] N.D. Birrell and P.C.W. Davies Quantum Fields in Curved Space, Cambridge University Press (1982) 
[16] G.N. Watson, A Treatise on the Theory of Bessel Functions, Cambridge University Press, (1966)

[17] S.W. Hawking, Commun. Math. Phys. 55, 133 (1977)

[18] F.D. Mazzitelli, J.P. Paz and M.A. Castagnino, Phys. Lett. B189, 132 (1987)

[19] G.N. Felder, A. Frolov, L. Kofman and A. Linde, Phys.Rev. D66, 023507 (2002)

[20] G.T. Horowitz and J. Polchinski, hep-th/0206228

[21] A.J. Tolley and N. Turok, hep-th/0204091

[22] H. Liu, G. Moore and N. Seiberg, hep-th/0204168 and hep-th/0206182; M. Fabinger and J. McGreevy, hep-th/0206196 